\title{
Hybrid finite element modelling of conformal antenna and array structures utilizing fast integral methods
}

\author{
T. F. Eibert, K. Sertel and J. L. Volakis*, ${ }^{\dagger}$ \\ Radiation Laboratory, Department of Electrical Engineering and Computer Science, University of Michigan, \\ Ann Arbor, MI 48109-2212, U.S.A.
}

\begin{abstract}
SUMMARY
Hybrid finite element methods (FEM) which combine the finite element and boundary integral methods have been found very successful for the analysis of conformal finite and periodic arrays embedded on planar or curved platforms. A key advantage of these hybrid methods is their capability to model inhomogeneous and layered material without a need to introduce complicated Green's functions. Also, they offer full geometrical adaptability and are thus of interest in general-purpose analysis and design. For the proposed hybrid FEM, the boundary integral is only used on the aperture to enforce the radiation condition by employing the standard free space Green's function.

The boundary integral truncation of the FEM volume domain, although necessary for rigor, is also the cause of substantial increase in CPU complexity. In this paper, we concentrate on fast integral methods for speeding-up the computation of these boundary integrals during the execution of the iterative solver. We consider both the adaptive integral method (AIM) and the fast multipole method (FMM) to reduce the complexity of boundary integral computation down to $\mathcal{O}\left(N^{\alpha}\right)$ with $\alpha<1.5$. CPU and memory estimates are given when the AIM and FMM accelerations are employed as compared to the standard $\mathcal{O}\left(N^{2}\right)$ algorithms. In addition, several examples are included to demonstrate the practicality and application of these fast hybrid methods to planar finite and infinite arrays, frequency selective surfaces, and arrays on curved platforms. Copyright (C) 2000 John Wiley \& Sons, Ltd.
\end{abstract}

\section{THREE-DIMENSIONAL FINITE ELEMENT-BOUNDARY INTEGRAL FORMULATION}

The finite element (FE)-boundary integral (BI) formulation for cavity backed antennas recessed in a ground plane has been given in References [1-3]. Below we briefly present the formulation with particular emphasis for modelling cavity backed antennas as well as periodic arrays. The differences between single elements and infinite periodic arrays are in the Green's function and in the necessity to apply periodic boundary conditions at the volume and surface boundaries to reduce the infinite periodic array to a single unit cell.

\footnotetext{
* Correspondence to: John L. Volakis, Radiation Laboratory, Department of Electrical Engineering and Computer Science, University of Michigan, Ann Arbor, MI 48109-2212, U.S.A.

${ }^{\dagger}$ E-mail: volakis@eecs.umich.edu
}

Copyright (C) 2000 John Wiley \& Sons, Ltd. 


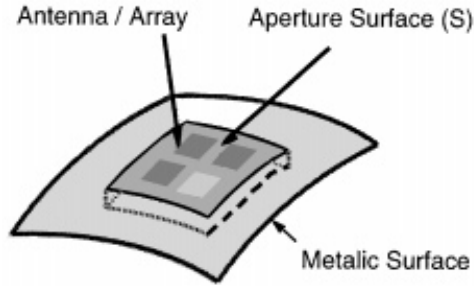

(a)

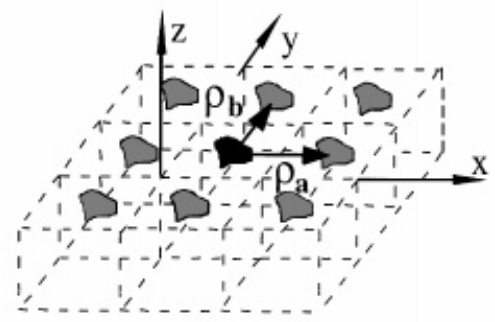

(b)

Figure 1. (a) Finite array in the cavity, (b) infinite periodic structure.

Let us consider the antenna/array structure illustrated in Figure 1. For time harmonic electromagnetic fields ( $\mathrm{e}^{\mathrm{j} \omega t}$ time convention), the weak form of the vector wave equation is [5]

$$
\begin{aligned}
\langle\mathbf{E}, \mathbf{T}\rangle= & \iiint_{V}\left[\frac{1}{\mu_{r}}(\nabla \times \mathbf{T}) \cdot(\nabla \times \mathbf{E})-\varepsilon_{r} k^{2}, \mathbf{E} \cdot \mathbf{T}+\mathrm{j} k Z_{0} \mathbf{T} \cdot \mathbf{J}^{\text {int }}\right] \mathrm{d} v \\
& +\mathrm{j} k Z \iint_{S} \mathbf{T} \cdot(\mathbf{H} \times \hat{n}) \mathrm{d} s
\end{aligned}
$$

where $\mathbf{T}$ is a weighting function, $\mathbf{J}^{\text {int }}$ denotes an excitation current within the FE domain, $S$ represents the boundaries of the FE domain, and $\hat{n}$ is the unit surface normal directed out of the FE domain. Also, $k$ and $Z$ are the free space wave number and wave impedance, respectively. The magnetic field $\mathbf{H}$ must be treated as an independent quantity over the boundary $S$ of the FE domain. It is, therefore, necessary to introduce an additional equation over $S$ for the unique solution of $\mathbf{E}$ and $\mathbf{H}$ over the volume boundaries. In the context of the FE-BI this is obtained by invoking the equivalence principle over $S$, allowing us to express $\mathbf{H}$ for a planar surface $S$ as

$$
\mathbf{H}=-2 j \frac{k}{Z}\left[\iint_{S} g\left(\mathbf{r}, \mathbf{r}^{\prime}\right)(\mathbf{E} \times \hat{n}) \mathrm{d} s+\frac{1}{k^{2}} \nabla \iint_{S} g\left(\mathbf{r}, \mathbf{r}^{\prime}\right) \nabla^{\prime} \cdot(\mathbf{E} \times \hat{n}) \mathrm{d} s\right]+\mathbf{H}^{\mathrm{inc}}
$$

where $g\left(\mathbf{r}, \mathbf{r}^{\prime}\right)$ is the scalar free space Green's function given as

$$
g\left(\mathbf{r}, \mathbf{r}^{\prime}\right)=\frac{1}{4 \pi} \frac{\mathrm{e}^{-\mathbf{j} k\left|\mathbf{r}-\mathbf{r}^{\prime}\right|}}{\left|\mathbf{r}-\mathbf{r}^{\prime}\right|}
$$

and $\mathbf{H}^{\mathrm{inc}}$ is an incident wave. For BI surfaces $S$ with small curvature, this explicit representation of $\mathbf{H}$ can be utilized to obtain an approximate solution whereas for BI surfaces with strong curvature a more general integral expression with implicit coupling of $\mathbf{E}$ and $\mathbf{H}$ must be used.

Equations (1) and (2) can now be discretized for a solution $\mathbf{E}$ within the volume cavity and on the aperture $S$.

To construct a linear set of equations from Equations (1) and (2), we must first tessellate the volume and introduce expansions for each of the tessellation elements. For meshing convenience, 


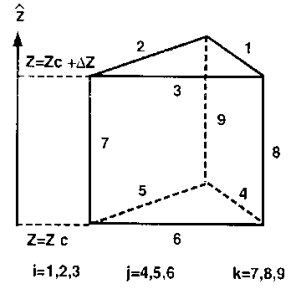

Figure 2. Right-angled prism.

we can choose tessellation elements that have constant height but must be more adaptable for surface modelling. The edge-based prismatic elements presented in References [2-4] allow for this type of flexibility. For doubly curved structures, distorted versions of the prismatic elements are employed whereas planar configurations and infinite periodic arrays can be discretized by the right-angled version as displayed in Figure 2. Using this type of tessellation elements (see Figure 2), the $\mathbf{E}$ field is expanded within the cavity volume as

$$
\mathbf{E}^{e}=\sum_{j=1}^{9} E_{j}^{e} \mathbf{W}_{j}^{e}=[\mathbf{W}]_{e}^{\mathrm{T}}\left\{E^{e}\right\}
$$

where $[\mathbf{W}]_{e}=\left[\left\{W_{x}\right\},\left\{W_{y}\right\},\left\{W_{z}\right\}\right]$ is explicitly given in Reference [3]. On the aperture, since the top and bottom faces of the prism are triangles, these are reduced to

where $[S]_{s}=\left[S_{x}, S_{y}\right]$ and

$$
\mathbf{E}^{s}(\mathbf{r})=\sum_{i=1}^{3} E_{i}^{s} \mathbf{S}_{i}^{s}(\mathbf{r})=[S]_{s}^{\mathrm{T}}\left\{E^{s}\right\}
$$

$$
\mathbf{S}_{i}=\frac{l_{i}}{2 A^{e}} \hat{z} \times\left(\mathbf{r}-\mathbf{r}_{i}\right)
$$

To generate a linear system for $E_{j}^{e}$, Equations (4) and (5) are substituted into Equations (1) and (2) and Galerkin's method (setting $\mathbf{T}=\mathbf{W}$ ) is employed to yield the assembled system

$$
[\mathscr{A}]\left\{\begin{array}{l}
\left\{E^{V}\right\} \\
\left\{E^{S}\right\}
\end{array}\right\}+\left[\begin{array}{cc}
{[0]} & {[0]} \\
{[0]} & {[\mathscr{B}]}
\end{array}\right]\left\{\begin{array}{l}
\left\{E^{V}\right\} \\
\left\{E^{S}\right\}
\end{array}\right\}=\left\{\begin{array}{l}
\left\{b^{V}\right\} \\
\left\{b^{S}\right\}
\end{array}\right\}
$$

In this system, $\left\{E^{V}\right\}$ denotes the field unknowns within the volume enclosed by $S$, whereas $\left\{E^{S}\right\}$ represents the corresponding unknowns on the boundary $S$. The excitation column $\left\{b^{V}\right\}$ is due to internal antenna sources and $\left\{b^{S}\right\}$ is associated with the incident field excitations (for scattering computations). When the above system refers to cavity backed antennas or finite arrays in a cavity backed configuration, the boundary condition on the vertical/side walls of the cavity is simply $\hat{n} \times \mathbf{E}=0$. This condition is implemented during the assembly process of the finite element system and results in the elimination of all unknowns associated with the metallic walls.

Infinite periodic arrays are assumed to be periodic in the $x y$-plane and the $(m, n)$ th cell of the array is obtained by shifting the $(0,0)$ th cell through the relation

$$
\boldsymbol{\rho}_{m n}=m \boldsymbol{\rho}_{a}+n \boldsymbol{\rho}_{b}
$$




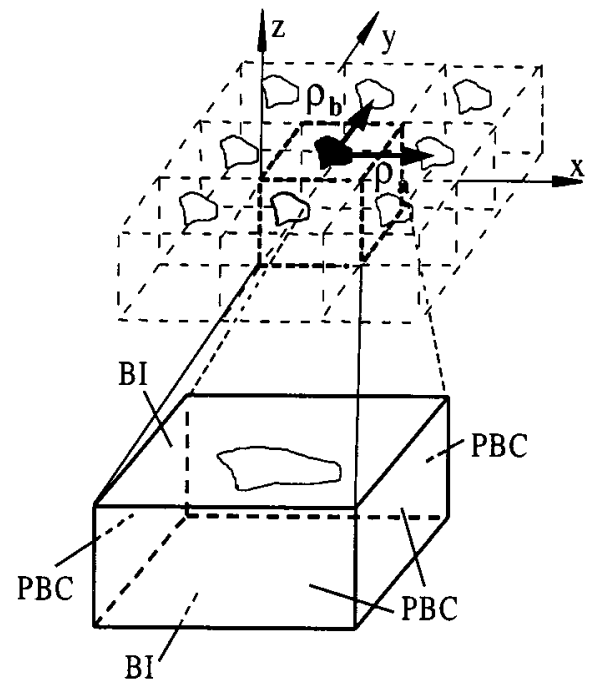

Figure 3. Reduction of infinite array to one unit cell.

Here, $\boldsymbol{\rho}_{a}, \boldsymbol{\rho}_{b}$ are the lattice vectors parallel to the $x y$-plane. For periodic excitation of the array with a linear phase factor, the fields in the array obey the periodicity conditions

where

$$
\mathbf{E}\left(\mathbf{r}+m \boldsymbol{\rho}_{a}+n \boldsymbol{\rho}_{b}\right)=\mathbf{E}(\mathbf{r}) \mathrm{e}^{-\mathbf{j} \mathbf{k}_{t 00} \cdot\left(m \boldsymbol{\rho}_{a}+n \boldsymbol{\rho}_{b}\right)}, \quad \mathbf{H}\left(\mathbf{r}+m \boldsymbol{\rho}_{a}+n \boldsymbol{\rho}_{b}\right)=\mathbf{H}(\mathbf{r}) \mathrm{e}^{-\mathbf{j} \mathbf{k}_{t 00} \cdot\left(m \boldsymbol{\rho}_{a}+n \boldsymbol{\rho}_{b}\right)}
$$

$$
\mathbf{k}_{t 00}=k_{x 00} \hat{\mathbf{x}}+k_{y 00} \hat{\mathbf{y}}=k \sin \vartheta_{0} \cos \varphi_{0} \hat{\mathbf{x}}+k \sin \vartheta_{0} \sin \varphi_{0} \hat{\mathbf{y}}
$$

with $\vartheta_{0}, \varphi_{0}$ describing the scan direction of the array or the propagation direction of an incident plane wave.

Using this periodicity condition and the boundary integral representation in (2), the computational domain can be reduced to a single unit cell of the array [6-8] (see Figure 3). This includes that in (2), $g\left(\mathbf{r}, \mathbf{r}^{\prime}\right)$ must be replaced by the appropriate periodic Green's function and the periodic boundary conditions (PBCs) must be explicitly enforced on the vertical side walls of the unit cell mesh.

In the spatial domain, the periodic Green's function $g_{p}\left(\mathbf{r}, \mathbf{r}^{\prime}\right)$ has the form [8]

$$
g_{p}\left(\mathbf{r}, \mathbf{r}^{\prime}\right)=\sum_{m=-\infty}^{\infty} \sum_{n=-\infty}^{\infty} \mathrm{e}^{-\mathrm{j} \mathbf{k}_{i 0} \cdot \boldsymbol{\rho}_{m n}} \frac{\mathrm{e}^{-\mathrm{j} k R_{m n}}}{4 \pi R_{m n}}
$$

where

$$
R_{m n}=\left|\mathbf{r}-\mathbf{r}^{\prime}-\boldsymbol{\rho}_{m n}\right|
$$

In the spectral domain, $g_{p}\left(\mathbf{r}, \mathbf{r}^{\prime}\right)$ becomes

$$
g_{\mathrm{p}}\left(\mathbf{r}, \mathbf{r}^{\prime}\right)=\frac{1}{A} \sum_{m=-\infty}^{\infty} \sum_{n=-\infty}^{\infty} \frac{1}{2 j k_{z m n}} \mathrm{e}^{-\mathrm{j} \mathbf{k}_{t m n} \cdot\left(\boldsymbol{\rho}-\boldsymbol{\rho}^{\prime}\right)} \mathrm{e}^{-\mathrm{j} k_{z m n}\left|z-z_{s}\right|}
$$


where $A=\left|\boldsymbol{\rho}_{a} \times \boldsymbol{\rho}_{b}\right|$ is the cross-sectional area of the unit cell,

$$
\begin{gathered}
\mathbf{r}=\boldsymbol{\rho}+z \hat{\mathbf{z}}, \\
\mathbf{k}_{t m n}=\mathbf{k}_{t 00}+\frac{2 \pi}{A}\left[m\left(\boldsymbol{\rho}_{b} \times \hat{\mathbf{z}}\right)+n\left(\hat{\mathbf{z}} \times \boldsymbol{\rho}_{a}\right)\right]
\end{gathered}
$$

is the so-called reciprocal lattice vector, and

$$
k_{z m n}=\sqrt{k^{2}-\mathbf{k}_{t m n} \cdot \mathbf{k}_{t m n}}
$$

where $\operatorname{Re}\left(k_{z m n}\right) \geqslant 0, \operatorname{Im}\left(k_{z m n}\right) \leqslant 0$. In many cases, the spectral domain representation (13) has satisfactory convergence behaviour if applied in a spectral-domain formulation of the integral equation $[6,7]$. However, for arbitrary array configurations analysed in the space domain, having strongly as well as weakly coupled array elements, it is necessary to have a representation that converges faster than either Equation (11) or Equation (13). This can be achieved by employing the so-called Ewald transformation originally proposed by Ewald for modelling optical and electrostatic potentials in three-dimensional ion lattices [15]. The Ewald transformation starts from the spatial domain representation of the periodic Green's function (11) and makes use of the identity

$$
\frac{\mathrm{e}^{-\mathrm{j} k R_{m n}}}{R_{m n}}=\frac{2}{\sqrt{\pi}} \int_{0}^{\infty} \mathrm{e}^{-R_{m n}^{2} s^{2}+\left(k^{2} / 4 s^{2}\right)} \mathrm{d} s
$$

where $s$ is a complex variable. In order that the integrand converges as $s \rightarrow 0$ for a wave number $k$ with an arbitrary amount of loss, the path is chosen so that $\arg (s)=\pi / 4$ as $s \rightarrow 0$. In order to have convergence as $s \rightarrow \infty$, the path is chosen so that $-\pi / 4 \leqslant \arg (s) \leqslant \pi / 4$. Next, Equation (17) is substituted into Equation (11) and the parameter $E$ is introduced to split the integral into two terms, as

$$
g_{p}\left(\mathbf{r}, \mathbf{r}^{\prime}\right)=g_{p 1}\left(\mathbf{r}, \mathbf{r}^{\prime}\right)+g_{p 2}\left(\mathbf{r}, \mathbf{r}^{\prime}\right)
$$

where

$$
\begin{aligned}
& g_{p 1}\left(\mathbf{r}, \mathbf{r}^{\prime}\right)=\frac{1}{4 \pi} \sum_{m=-\infty}^{\infty} \sum_{n=-\infty}^{\infty} \mathrm{e}^{-\mathbf{j} \mathbf{k}_{t 0} \cdot \mathbf{\rho}_{m n}} \frac{2}{\sqrt{\pi}} \int_{0}^{E} \mathrm{e}^{-R_{m n}^{2} s^{2}+\left(k^{2} / 4 s^{2}\right)} \mathrm{d} s \\
& g_{p 2}\left(\mathbf{r}, \mathbf{r}_{s}\right)=\frac{1}{4 \pi} \sum_{m=-\infty}^{\infty} \sum_{n=-\infty}^{\infty} \mathrm{e}^{-\mathrm{j} \mathbf{k}_{t 0} \cdot \boldsymbol{\rho}_{m n}} \frac{2}{\sqrt{\pi}} \int_{E}^{\infty} \mathrm{e}^{-R_{m n}^{2} s^{2}+\left(k^{2} / 4 s^{2}\right)} \mathrm{d} s
\end{aligned}
$$

Upon applying some analytical manipulations $[8,14,15]$, the two series can finally be written as

$$
\begin{aligned}
g_{p 1}\left(\mathbf{r}, \mathbf{r}^{\prime}\right)= & \frac{1}{A} \sum_{m=-\infty}^{\infty} \sum_{n=-\infty}^{\infty} \frac{1}{4 j k_{z m n}} \mathrm{e}^{-\mathrm{j} \mathbf{k}_{t m n} \cdot\left(\boldsymbol{\rho}-\boldsymbol{\rho}^{\prime}\right)}\left[\mathrm{e}^{-\mathrm{j} k_{z m n}\left|z-z^{\prime}\right|} \operatorname{erfc}\left(\frac{\mathrm{j} k_{z m n}}{2 E}-\left|z-z^{\prime}\right| E\right)\right. \\
& \left.+\mathrm{e}^{\mathrm{j} k_{z m n}\left|z-z^{\prime}\right|} \operatorname{erfc}\left(\frac{\mathrm{j} k_{z m n}}{2 E}+\left|z-z^{\prime}\right| E\right)\right]
\end{aligned}
$$


and

$$
\begin{aligned}
g_{p 2}\left(\mathbf{r}, \mathbf{r}^{\prime}\right)= & \sum_{m=-\infty}^{\infty} \sum_{n=-\infty}^{\infty} \frac{\mathrm{e}^{-\mathbf{j} \mathbf{k}_{t 00} \cdot \mathbf{p}_{m n}}}{8 \pi R_{m n}}\left[\mathrm{e}^{-\mathrm{j} k_{0} R_{m n}} \operatorname{erfc}\left(R_{m n} E-\frac{\mathrm{j} k}{2 E}\right)\right. \\
& \left.+\mathrm{e}^{\mathrm{j} k \boldsymbol{R}_{m n}} \operatorname{erfc}\left(R_{m n} E+\frac{\mathrm{j} k}{2 E}\right)\right]
\end{aligned}
$$

where erfc is the complementary error function. Equation (22) is essentially a 'modified' spatial domain portion of the periodic Green's function and Equation (21) can be identified as a 'modified' spectral domain portion of the periodic Green's function. The two expressions (22) and (21) both converge exponentially (Gaussian convergence) and their computation is therefore very efficient requiring only a few terms of the series. The parameter $E$ controls the convergence rate. As $E$ becomes larger, the spatial series (22) converges faster, while the spectral series (21) converges slower. The optimum parameter is that which makes the two series converge at the same rate, so that equal numbers of terms are required in the calculation of both series (assuming the same calculation time for corresponding terms in each of the two series). By analysis of the asymptotic behaviour of the series terms, the optimum parameter $E_{\mathrm{opt}}$ is found to be [14]

$$
E_{\mathrm{opt}}=\sqrt{\frac{\pi}{A}}
$$

Choosing this value for $E$ and adjusting the summation limits so that the most dominant terms are kept, in almost all practical cases it is sufficient to include only 9 summation terms in Equations (22) and (21) (i.e., the summation limits are from -1 to +1 ), in which case the error level is usually less than 0.1 per cent.

\section{FINITE ELEMENT-FAST INTEGRAL METHODS}

\subsection{Introduction}

The FE-BI system (7) is partly sparse and partly dense. More specifically, [ $\mathscr{A}]$ is sparse, whereas $[\mathscr{B}]$ is dense. Thus, although $[\mathscr{B}]$ is much smaller in rank than $[\mathscr{A}]$, it is usually responsible for most of the CPU and memory requirements when an iterative algorithm is used for the solution of Equation (7). To alleviate the CPU and memory needs, fast integral (FI) methods have recently been introduced [16-19] to perform the matrix-vector product $[\mathscr{B}]\left\{E^{S}\right\}$ faster and using less memory. Two of these approaches are the adaptive integral method (AIM) [17], and the fast multipole method (FMM) [18,19]. Both FMM and AIM reduce the CPU time and memory requirement from $\mathcal{O}\left(N^{2}\right)$ down to $\mathcal{O}\left(N^{\alpha}\right)$ where $\alpha \leqslant 1.5$. The main feature of AIM and FMM is the decomposition of the matrix as

$$
[\mathscr{B}]=[\mathscr{B}]^{\text {near }}+[\mathscr{B}]^{\text {far }}
$$

based on some threshold distance referred to as the near-zone radius. The matrix $[\mathscr{B}]^{\text {near }}$ contains the interactions between elements separated less than the threshold distance, whereas $[\mathscr{B}]^{\mathrm{far}}$ contains the remaining interactions. The elements of $[\mathscr{B}]^{\text {near }}$ are evaluated without approximation. However, the product $[\mathscr{B}]^{\mathrm{far}}\left\{E^{S}\right\}$ is evaluated in an approximate manner leading to a much 
faster execution. FMM achieves its CPU reduction by grouping the far-zone unknowns and interacting their weighted contributions. In the case of AIM, the CPU reduction is achieved by mapping the original method of moments $(\mathrm{MoM})$ discretization onto a uniform rectangular grid and exploiting the Toeplitz property of the Green's function on this grid. That is, the fast Fourier transform (FFT) and the convolution theorem are invoked to compute the matrix-vector products in an iterative solver. Next, we describe how the evaluation of $[\mathscr{B}]\left\{E^{S}\right\}$ is performed for each of the fast algorithms.

\subsection{Adaptive integral method}

2.2.1 Theory and implementation. The basic idea of AIM is to split [ $\mathscr{B}]$ in (24) as

$$
[\mathscr{B}]=[\mathscr{B}]^{\text {near }}+[\mathscr{B}]^{\text {far }}
$$

where $[\mathscr{B}]^{\text {near }}$ contains the elements of $[\mathscr{B}]$ that are near the self-cell and correspondingly $[\mathscr{B}]^{\text {far }}$ contains the remaining 'far-zone' elements of $[\mathscr{B}]$. AIM reduces the CPU time and memory of the iterative solver by exploiting convolutional properties of the Green's function for the evaluation of the matrix-vector products associated with the mostly full matrix $[\mathscr{B}]^{\mathrm{far}}$. That is, the far-zone matrix is not explicitly generated and the matrix-vector products are performed in the discrete Fourier domain (DFT) utilizing appropriate 2D-FFT algorithms [20]. However, the convolutional properties can only be exploited on a uniform grid and therefore, the basis functions on the original and usually irregular triangular mesh must first be mapped onto a uniform grid. To do so, we introduce auxiliary basis functions on a uniform rectangular grid which is coincident with the original triangular grid (see Figure 4). The auxiliary expansion for the magnetic surface currents (equivalent to the tangential electric field intensities on the top and bottom BI surfaces of

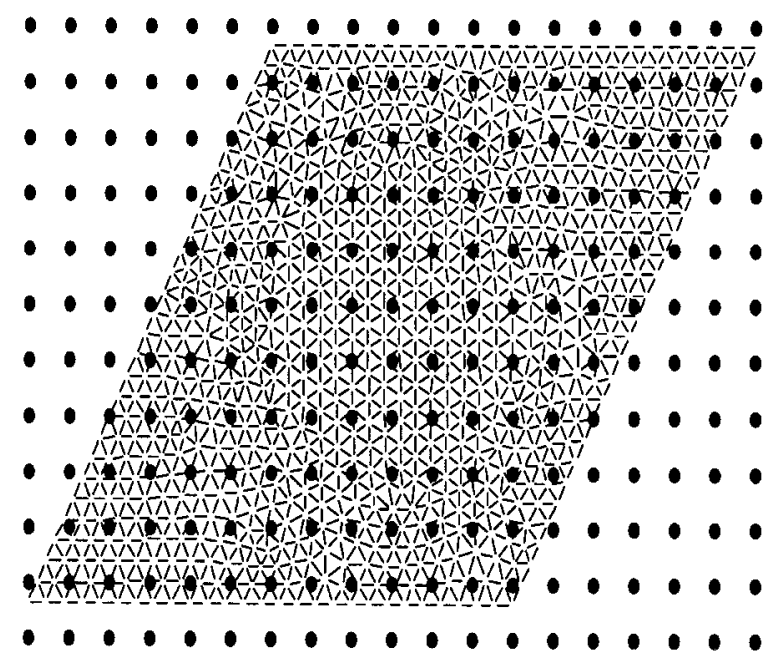

Figure 4. Uniform AIM grid overlaying arbitrarily shaped irregular mesh. 
the unit cell) is given by

$$
\begin{aligned}
\mathbf{M}_{s}^{\mathrm{aux}} & =\sum_{i=0}^{I-1} \sum_{j=0}^{J-1}\left[M_{i j}^{x} \hat{x}+M_{i j}^{y} \hat{y}\right] \delta\left(x-x_{0}-\mathrm{i} \Delta x\right) \delta\left(y-y_{0}-\mathrm{j} \Delta y\right) \\
& =\sum_{n=1}^{N} M_{n} \sum_{i=0}^{I-1} \sum_{j=0}^{J-1}\left[\Lambda_{i j}^{x, n} \hat{x}+\Lambda_{i j}^{y, n} \hat{y}\right] \delta\left(x-x_{0}-\mathrm{i} \Delta x\right) \delta\left(y-y_{0}-\mathrm{j} \Delta y\right)
\end{aligned}
$$

where $\left(x_{0}, y_{0}\right)$ is the lower left corner of the uniform grid and $\Delta x, \Delta y$ are the sample distances in the $x$ and $y$ directions, respectively. $M_{i j}^{x}, M_{i j}^{y}$ are the expansion coefficients on the uniform grid, $M_{n}$ are the expansion coefficients on the original mesh, and $\Lambda_{i j}^{x, n}, \Lambda_{i j}^{y, n}$ are the equivalent expansion coefficients on the uniform grid for each single expansion function on the original mesh. Since we work with a mixed potential integral equation (MPIE) formulation for the BI implementation, we introduce the additional expansion

$$
\begin{aligned}
\nabla_{s} \cdot \mathbf{M}_{s}^{\mathrm{aux}} & =\sum_{i=0}^{I-1} \sum_{j=0}^{J-1} Q_{i j} \delta\left(x-x_{0}-\mathrm{i} \Delta x\right) \delta\left(y-y_{0}-\mathrm{j} \Delta y\right) \\
& =\sum_{n=1}^{N} Q_{n} \sum_{i=0}^{I-1} \sum_{j=0}^{J-1} \Lambda_{i j}^{Q, n} \delta\left(x-x_{0}-\mathrm{i} \Delta x\right) \delta\left(y-y_{0}-\mathrm{j} \Delta y\right)
\end{aligned}
$$

for the surface divergence of the surface currents.

To find an appropriate relationship between the uniform expansion (26) and (27) and the basis functions on the original possibly irregular triangular mesh for evaluation of the far interactions, we start with the common MPIE expression [9]

$$
\begin{aligned}
\mathscr{B}_{m n}= & \mathscr{B}_{m n}^{(1)}+\mathscr{B}_{m n}^{(2)} \\
= & -2 k^{2} \iint_{S_{m}} \iint_{S_{n}} g_{p}\left(\mathbf{r}, \mathbf{r}_{s}\right) \mathbf{f}_{m}(\mathbf{r}) \cdot \mathbf{f}_{n}\left(\mathbf{r}_{s}\right) \mathrm{d} S_{s} \mathrm{~d} S \\
& +2 \iint_{S_{m}} \iint_{S_{n}} g_{p}\left(\mathbf{r}, \mathbf{r}_{s}\right), \nabla_{s} \cdot \mathbf{f}_{m}(\mathbf{r}) \nabla_{s} \cdot \mathbf{f}_{n}\left(\mathbf{r}_{s}\right) \mathrm{d} S_{s} \mathrm{~d} S
\end{aligned}
$$

for the calculation of an arbitrary coupling element for the original expansion. Here, $\mathbf{f}_{n}$ and $\mathbf{f}_{m}$ are the source and testing basis functions on the triangles with the areas $S_{n}$ and $S_{m}$, respectively. Also, the expression is given for a periodic Green's function $g_{p}\left(\mathbf{r}, \mathbf{r}^{\prime}\right)$. With the definitions

and

$$
q_{m}(\mathbf{r})=\nabla_{s} \cdot \mathbf{f}_{m}(\mathbf{r}), \quad q_{n}\left(\mathbf{r}^{\prime}\right)=\nabla_{s} \cdot \mathbf{f}_{n}\left(\mathbf{r}^{\prime}\right)
$$

$\mathscr{B}_{m n}^{(2)}$ can be written as

$$
g(\mathbf{r})=\iint_{S_{n}} g_{p}\left(\mathbf{r}, \mathbf{r}^{\prime}\right) q_{n}\left(\mathbf{r}^{\prime}\right) \mathrm{d} S_{s}
$$

$$
\mathscr{B}_{m n}^{(2)}=2 \iint_{S_{m}} q_{m}(\mathbf{r}) g(\mathbf{r}) \mathrm{d} S
$$


Without loss of generality, the evaluation of the coupling integrals can be performed in the plane $z=0$. Based on this observation and introducing the Taylor series expansion

$$
g(\mathbf{r})=\sum_{q=q_{1}+q_{2}=0}^{\infty} a_{q}\left(x-x_{1}\right)^{q_{1}}\left(y-y_{1}\right)^{q_{2}}
$$

around the expansion point $\mathbf{r}=\left(x_{1}, y_{1}\right)$ in the plane $z=0, \mathscr{B}_{m n}^{(2)}$ can be written in the form

$$
\mathscr{B}_{m n}^{(2)}=2 \sum_{q=q_{1}+q_{2}=0}^{\infty} a_{q} \iint_{S_{m}} q_{m}(\mathbf{r})\left(x-x_{1}\right)^{q_{1}}\left(y-y_{1}\right)^{q_{2}} \mathrm{dS}
$$

which is equivalent to summing up the moments of the testing function $q_{m}$ multiplied by the Taylor coefficients $a_{q}$. In principle, the expansion point $\left(x_{1}, y_{1}\right)$ can be chosen arbitrarily but it is numerically advantageous to put it at the centre of the edge for which $q_{m}$ is defined [17]. Based on Equation (33), $\mathscr{B}^{(2)}$ can be calculated exactly by employing the equivalent basis functions on the uniform grid if the moments of the equivalent basis functions are equal to the moments of $q_{m}$. So, in principle, the equivalent amplitudes $\Lambda_{i j}^{Q, m}$ for the $m$ th basis function on the uniform grid can be obtained by enforcing the equality

$$
\begin{aligned}
\iint_{S_{m}} q_{m}(\mathbf{r})\left(x-x_{1}\right)^{q_{1}}\left(y-y_{1}\right)^{q_{2}} \mathrm{~d} S & =\sum_{i=0}^{I-1} \sum_{j=0}^{J-1} \Lambda_{i j}^{Q, m}\left(x_{0}+\mathrm{i} \Delta x-x_{1}\right)^{q_{1}}\left(y_{0}+\mathrm{j} \Delta y-y_{1}\right)^{q_{2}}, \\
q & =q_{1}+q_{2}=0, \ldots, \infty
\end{aligned}
$$

where the filter property of the $\delta$-functions was utilized. In a numerical implementation, only a finite number of moments can be enforced to be equal. However, if the distance between source and test subdomains is not too small, the function $g(\mathbf{r})$ is well behaved and an accurate evaluation of the coupling integrals can be achieved with a small number of the lowest-order moments. We restrict ourselves to $3 \times 3=9$ moments, so that each basis function $q_{m}$ must only be related to nine basis functions of the uniform grid. That is, Equation (34) is evaluated for indices $i=\left(i_{m}-1\right), \ldots,\left(i_{m}+1\right)$ and $j=\left(j_{m}-1\right), \ldots,\left(j_{m}+1\right)$, where $i_{m}$ and $j_{m}$ are the indices of the uniform grid point which is closest to the centre of edge $m$. The resulting $9 \times 9$ linear algebraic system can then be solved for the nine $\Lambda_{i j}^{Q, m}$ coefficients. The approximate expression for $\mathscr{B}_{m n}^{(2)}$ can be finally written as

$$
\mathscr{B}_{m n}^{(2)} \approx 2 \sum_{i=\left(i_{m}-1\right)}^{\left(i_{m}+1\right)} \sum_{j=\left(j_{m}-1\right)}^{\left(j_{m}+1\right)} g\left(x_{0}+\mathrm{i} \Delta x, y_{0}+\mathrm{j} \Delta y\right) \Lambda_{i j}^{Q, m}
$$

where again the filter property of the $\delta$-functions in Equation (27) was utilized. A similar procedure can be employed for the source integral in Equation (30). For this purpose, a Taylor series expansion of the Green's function $g_{p}$ is introduced with respect to the source point $\mathbf{r}^{\prime}$ for a fixed observation point $\mathbf{r}$. The obtained approximate expression for $g$ is

$$
g(\mathbf{r}) \approx \sum_{k=\left(k_{n}-1\right)}^{\left(k_{n}+1\right)} \sum_{l=\left(l_{n}-1\right)}^{\left(l_{n}+1\right)} g_{p}\left(\mathbf{r},\left(x_{0}+k \Delta x, y_{0}+l \Delta y\right)\right) \Lambda_{k l}^{Q, n}
$$

where $k_{n}, l_{n}$ are the indices of the closest uniform grid point with respect to the centre of edge $n$ and $\Lambda_{k l}^{Q, n}$ are the equivalent amplitudes for the respective basis functions on the uniform grid for basis 
function $n$ on the original irregular mesh. The combination of (35) and (36) results in

$$
\mathscr{B}_{m n}^{(2)} \approx 2 \sum_{i=\left(i_{m}-1\right)}^{\left(i_{m}+1\right)} \sum_{j=\left(j_{m}-1\right)}^{\left(j_{m}+1\right)} \sum_{k=\left(k_{n}-1\right)}^{\left(k_{n}+1\right)} \sum_{l=\left(l_{n}-1\right)}^{\left(l_{n}+1\right)} \Lambda_{k l}^{Q, n} g_{p}((i \Delta x, j \Delta y),(k \Delta x, l \Delta y)) \Lambda_{i j}^{Q, m}
$$

in which $x_{0}, y_{0}$ were omitted because of the shift invariance of $g_{p}$. In matrix form, we can write

$$
[\mathscr{B}]^{(2)} \approx 2[\Lambda]_{Q}\left[g_{p}\right][\Lambda]_{Q}^{\mathrm{T}}
$$

Expression (38) represents a matrix product of the two sparse $\Lambda$-matrices and the fully populated Green's function matrix $\left[g_{p}\right]$ of Toeplitz form. $\mathscr{B}_{m n}^{(1)}$ in $(28)$ is evaluated in a similar way as $\mathscr{B}_{m n}^{(2)}$ except that the $x$ and $y$ components of the magnetic currents must be considered. Therefore, the resulting representation for $\mathscr{B}_{m n}$ is

$$
[\mathscr{B}] \approx[\mathscr{B}]_{\mathrm{AIM}}=-2 k^{2}\left([\Lambda]_{x}\left[g_{p}\right][\Lambda]_{x}^{\mathrm{T}}+[\Lambda]_{y}\left[g_{p}\right][\Lambda]_{y}^{\mathrm{T}}\right)+2[\Lambda]_{Q}\left[g_{p}\right][\Lambda]_{Q}^{\mathrm{T}}
$$

where $[\Lambda]_{x}$ and $[\Lambda]_{y}$ are sparse matrices containing the equivalent current amplitudes introduced in Equation (26).

For the implementation of AIM, it is assumed that $[\mathscr{B}]_{\text {AIM }}$ is sufficiently accurate for the far-zone elements. For the near-zone interactions we keep the original matrix elements. Therefore, we decompose the AIM matrix as

$$
[\mathscr{B}]_{\text {AIM }}=[\mathscr{B}]_{\text {AIM }}^{\text {near }}+[\mathscr{B}]_{\text {AIM }}^{\text {far }}
$$

and when this is combined with Equation (25), we can rewrite the original $\mathscr{B}$ matrix as

$$
[\mathscr{B}]^{\text {approx }}=\left([\mathscr{B}]^{\text {near }}-[\mathscr{B}]_{\text {AIM }}^{\text {near }}\right)+[\mathscr{B}]_{\text {AIM }} \text {. }
$$

With this representation of $[\mathscr{B}]$, the near-zone interactions are evaluated without compromise in accuracy. However, since the majority of $[\mathscr{B}]^{\text {approx }}$ consists of the Toeplitz kernel $\left[g_{p}\right]$, the associated matrix-vector products can be performed using only $\mathcal{O}\left(n_{s}\right)$ memory and $\mathcal{O}\left(n_{s} \log n_{s}\right)$ CPU time. In the final numerical implementation, a near-zone threshold is defined so that $[\mathscr{B}]^{\text {approx }}$ is a sufficiently accurate representation of $[\mathscr{B}]$. The threshold distance is mostly controlled by the quasi-static singularities of the Green's function. In the case of the infinite periodic Green's function, we must also account for image singularities in the neighbouring periodic cells since they can be close to the test subdomains in the unit cell.

For calculation of matrix-vector products in an iterative solver, $[\mathscr{B}]_{\text {AIM }}$ is not computed explicitly. After mapping the actual source distributions onto the uniform grid through the $\Lambda$ matrices, the pertinent matrix-vector products are performed in the DFT domain using an FFT algorithm for the corresponding transformations. After transformation of the results back to the spatial domain, the fields on the original mesh are obtained by reverse mapping between the auxiliary unknowns and the original grid unknowns. However, for a computation of $[\mathscr{B}]_{\text {AIM }}^{\text {near }}$, it is advantageous to collect the contributions of the uniform grid in the spatial domain before the Toeplitz Green's function is transformed into the DFT domain. 


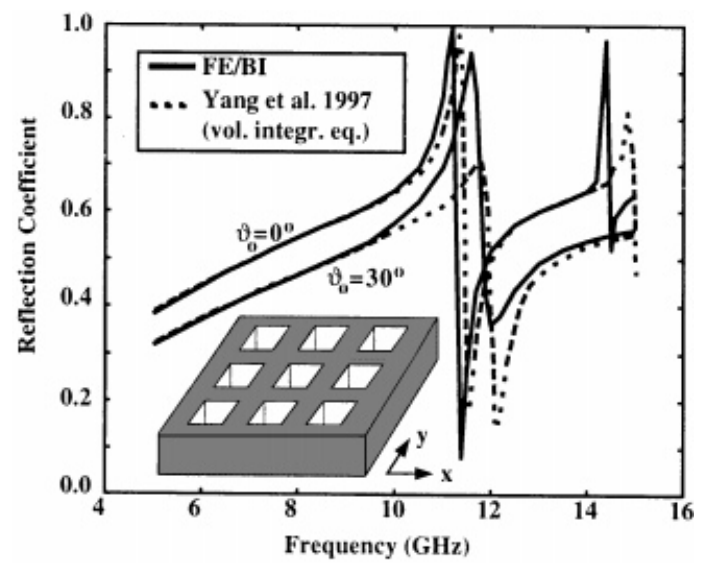

Figure 5. TM Plane wave reflection from a dielectric slab $\left(\varepsilon_{r}=4\right)$ with planarly embedded periodic material blocks $\left(\varepsilon_{r}=10\right)$ compared to reference values from [10]. Slab height: $0.2 \mathrm{~cm}$, period: $2 \times 2 \mathrm{~cm}$, block side length: $1 \times 1 \mathrm{~cm}, \varphi_{0}=0^{\circ}$.

\subsubsection{Applications}

Photonic bandgap materials. As an example we consider the dielectric slab in Figure 5 with embedded periodic material blocks. These lattices are often referred to as photonic bandgap materials. The diagram in Figure 5 shows the reflection coefficient of plane waves incident on the slab with different angles of incidence. The reflection coefficient curves exhibit the typical resonances of photonic bandgap materials. Compared to calculations obtained by a volume integral equation method [10], the first resonance is slightly shifted to a lower frequency whereas the frequency shift for the second resonance is larger. For TM-waves with oblique incidence, the resonances shift to higher frequencies, in agreement with Reference [10].

Artifical puck plate bandpass structure. Figure 6 shows the unit cell and the employed surface mesh for the so-called 'artificial puck plate' FSS screen which was presented in Reference [11] and analysed in Reference [6]. The basic FSS element is a dielectric-filled cylindrical waveguide with metallic walls and circular metallic irises in its apertures. On the top and bottom of the metallic plate, dielectric layers are placed for the optimization of the frequency behaviour of the bandpass structure. Our calculations are given in Figure 7 and are compared to MoM data and FE/BI results based on a tetrahedral mesh published in Reference [6]. As can be seen, our FE/BI curves are closer to the MoM curves than the FE/BI results from Reference [6]. This is likely due to our higher mesh density.

Noncommensurate multilayer frequency selective surfaces. Figure 8 gives the geometries and results for an eight-layer low-pass FSS structure (normal incidence) as well as for a five-layer configuration (oblique incidence). Both structures have the non-commensurate periodicities as given in the figure. Since non-commensurate periods cannot be handled in a straightforward manner approximate FE/BI models were used which assume that the fields in the array structure obey the varying geometrical periodicities. The FE/BI results are compared to data published in Reference [12]. For the eight-layer FSS, the FE/BI curve is closer to the measured results than the MoM curve. However, the oscillations of the transmission factor in the pass-band are less pronounced than for both reference results from Reference [12]. 

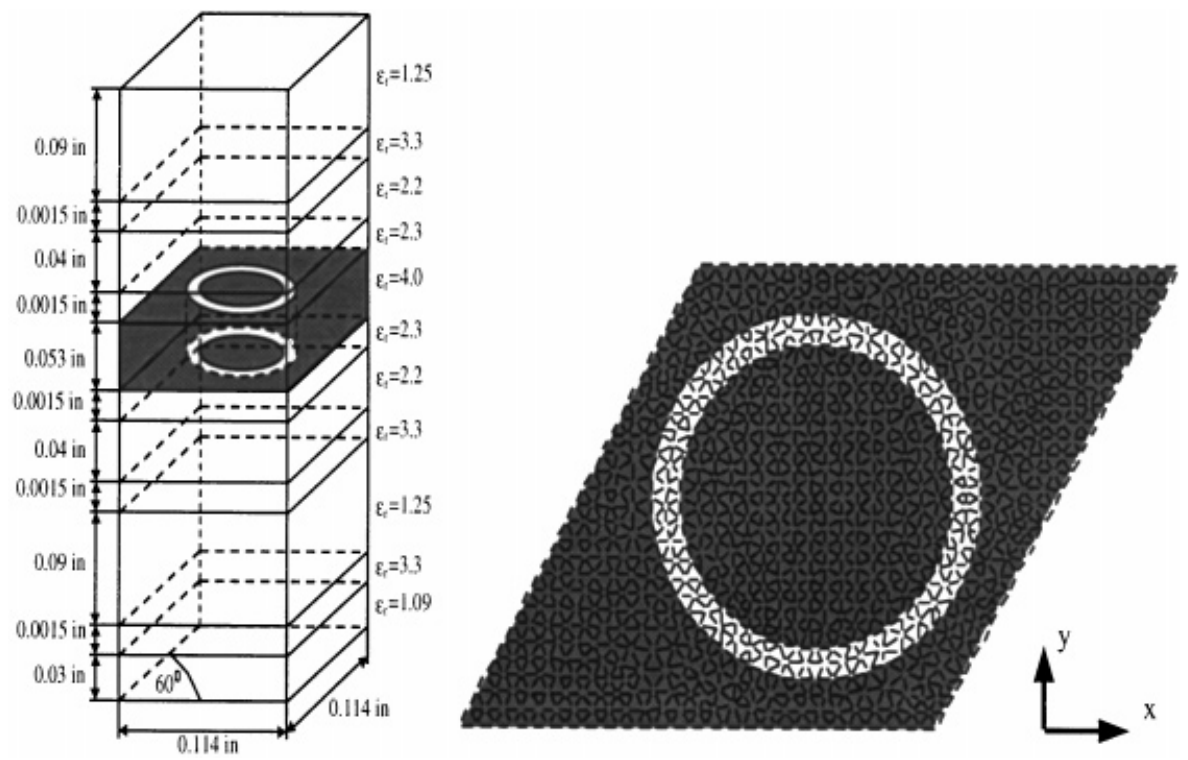

Figure 6. Geometry and mesh for unit cell of 'artificial puck plate' FSS as presented in Reference [6].

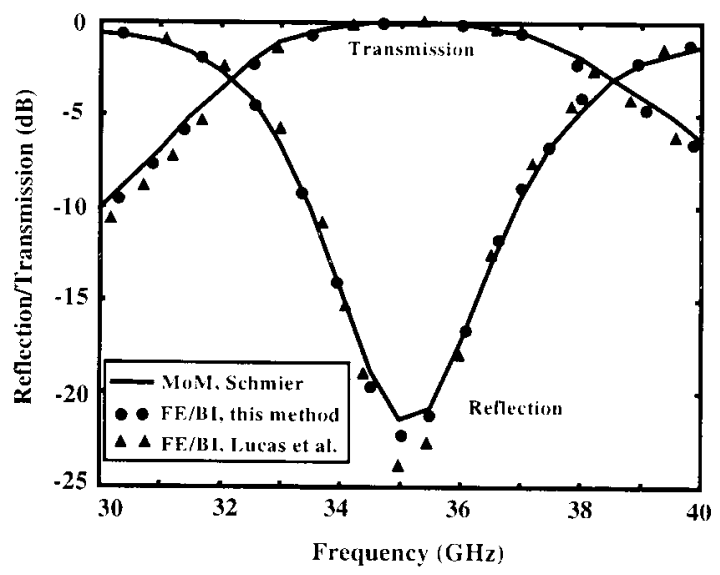

Figure 7. TE Reflection and transmission coefficients for bandpass structure in Figure 6 compared to reference values from Reference [6]. $\vartheta_{0}=0^{\circ}, \varphi_{0}=0^{\circ}$.

The transmission curves for the five-layer configuration in Figure $8(\mathrm{~b})$ were obtained for oblique incidence with $\vartheta_{0}=70^{\circ}, \varphi_{0}=0^{\circ}$. Results are given for TE as well as TM polarization. For the $\mathrm{TM}$ case, the FE/BI results agree very well with MoM results from Reference [12]; however, in the TE case, the oscillations in the pass-band of the FE/BI results are again less pronounced than in the MoM data. 


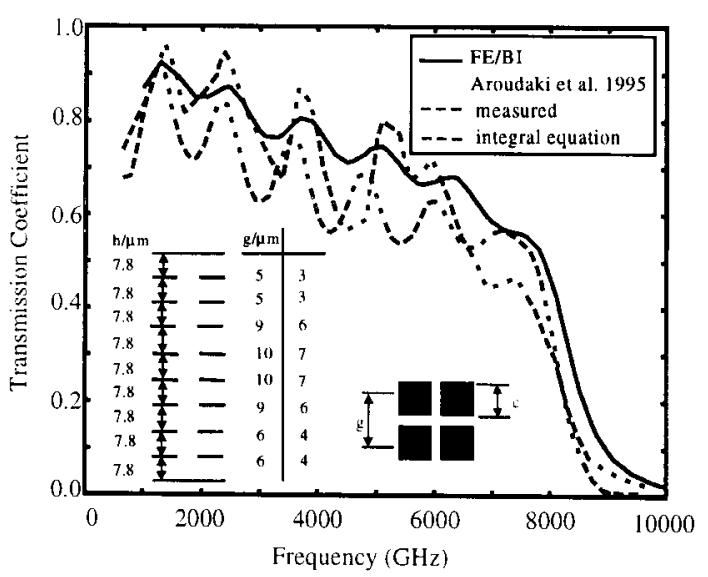

(a)

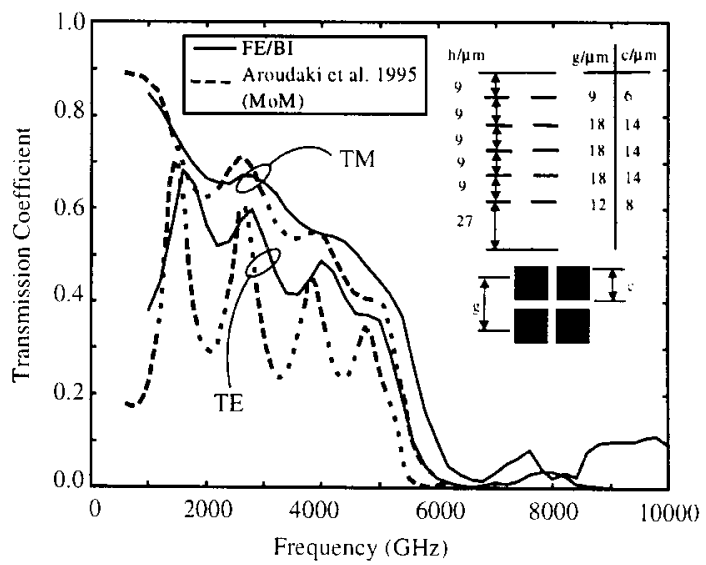

(b)

Figure 8. (a) Transmission coefficient of eight-layer FSS for normal incidence $\left(\varepsilon_{r}=2.3-j 0.08\right)$, (b) transmission coefficient of five-layer FSS for oblique incidence $\left(\vartheta_{0}=70^{\circ}, \varphi_{0}=0^{\circ}, \varepsilon_{r}=2.3-j 0.08\right)$.

2.2.3. CPU timings and memory requirements. To illustrate the memory and CPU time savings of the AIM acceleration, we analysed different mesh configurations of a microstrip dipole array. All meshes consisted of one volume prism along the depth of the array and a metallic backing on the bottom surface. Different meshes with increasing numbers of BI unknowns were generated by grouping several array elements into the discretized unit cell. The largest mesh was a $4 \times 4$ array and had 18208 BI unknowns (24448 volume mesh unknowns). The operation frequency was close to the half-wave resonance of the dipole elements. The density of the uniform AIM grid on the BI surface in the top of the unit cell mesh was chosen so that on average approximately 3.5 uniform grid points were placed per triangle side. The near-zone threshold was set so that the original matrix elements were used within 15 uniform grid samples in the $x$ and $y$ directions around the source element centre. The near-zone threshold was kept constant for all frequencies. In Figure 9, the number of matrix elements in the system matrix dependent on the number of BI unknowns is depicted as a measure of the memory requirements of the algorithm (12 bytes per matrix element for single precision and sparse matrix storage). In the conventional BI formulation, the number of matrix elements increases with complexity $\mathcal{O}\left(n_{s}^{2}\right)$ whereas AIM results in an optimal complexity of $\mathcal{O}\left(n_{s}\right)$. Due to the relatively expensive series representation of the periodic Green's function, the most time-consuming portion of our FE/BI approach was the BI fill time which is given in Figure 10(a) together with the total solution time. It can be seen that the AIM acceleration reduces the complexity of the BI fill from about $\mathcal{O}\left(n_{s}^{2}\right)$ to about $\mathcal{O}\left(n_{s}\right)$ (constant number of terms in the series representation of the Green's function assumed for both values). The complexity of the CPU time per iteration for the conventional and AIM-accelerated BI approaches is depicted in Figure 10(b). Results are given for the biconjugate gradient (BiCG) and generalized minimal residual (GMRES) solvers. In all cases, the GMRES solver (restarted every 50 iterations) needs approximately half the CPU time required by the BiCG solver since the GMRES solver needs only one matrix-vector product per iteration whereas the BiCG solver needs two matrix-vector products. As illustrated in Figure 10(b), the AIM acceleration reduces the CPU time complexity per 


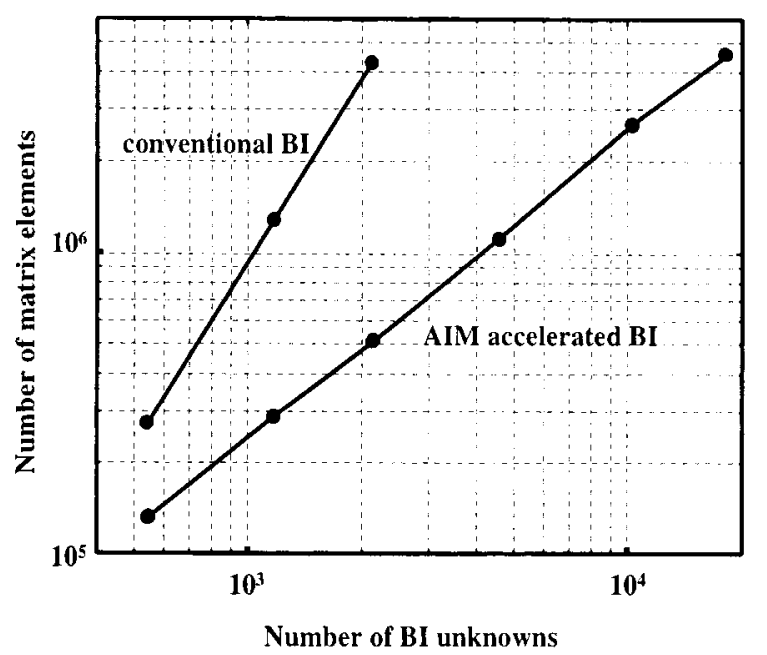

Figure 9. Number of matrix elements in the system matrix (FE and BI near-zone).

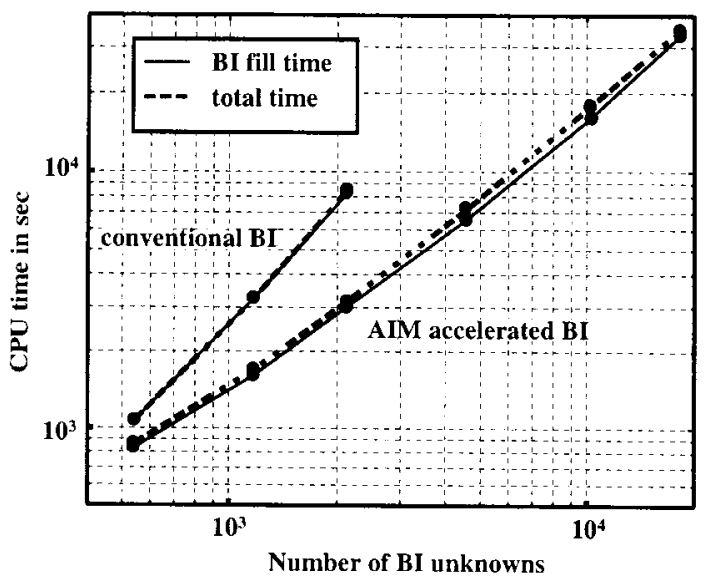

(a)

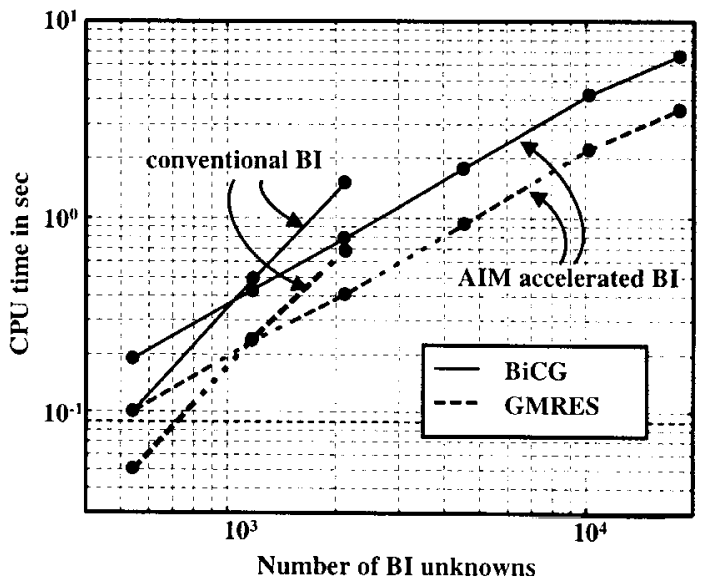

(b)

Figure 10. (a) BI fill time and total solution time for test problem of microstrip dipole array, (b) CPU time per iteration for test problem of microstrip dipole array.

matrix-vector product from $\mathcal{O}\left(n_{s}^{2}\right)$ to $\mathcal{O}\left(n_{s} \log n_{s}\right)$. However, the necessary AIM overhead leads to increased $\mathrm{CPU}$ time requirements for very small numbers of unknowns.

\subsection{Fast multipole method}

2.3.1. Theory and implementation. The FMM [16,18,19] is based on two elementary identities. One of them is the expansion of the scalar Green's function appearing in moment 


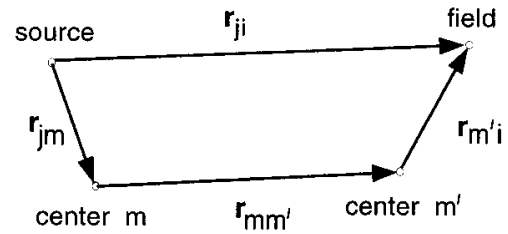

Figure 11. The geometry constructions used in FMM formulations illustrating the relation between source point, field point and the group centres.

matrix elements as [23]

$$
\frac{\mathrm{e}^{-\mathrm{j} k|\mathbf{r}+\mathbf{d}|}}{|\mathbf{r}+\mathbf{d}|}=-\mathrm{j} k \sum_{l=0}^{\infty}(-1)^{l}(2 l+1) j_{l}(k d) h_{l}^{(2)}(k r) P_{l}(\hat{d} \cdot \hat{r})
$$

Here $j_{l}$ is the spherical Bessel function, $h_{l}^{(2)}$ is the spherical Hankel function of the second kind, $P_{l}$ is the Legendre polynomial, and $d<r$ is the condition for the validity of the expansion. In the FMM formulations, where the source point is denoted by $\mathbf{x}^{\prime}$ and the observation point by $\mathbf{x}, \mathbf{r}$ will be chosen to be close to $\mathbf{x}-\mathbf{x}^{\prime}$ so that $\mathbf{d}$ will be small as depicted in Figure 11 . The second identity is the expansion of the product $j_{l} P_{l}$ appearing in Equation (42) as a sum of propagating plane waves (spectral integral):

$$
(-1)^{l} 4 \pi j^{l} j_{l}(k d) P_{l}(\hat{d} \cdot \hat{r})=\int^{2} \hat{k} \mathrm{e}^{-\mathbf{j k} \cdot \mathbf{d}} P_{l}(\hat{k} \cdot \hat{r})
$$

Using this identity, expansion (42) can be rewritten as

$$
\frac{\mathrm{e}^{-\mathrm{j} k|\mathbf{r}+\mathbf{d}|}}{|\mathbf{r}+\mathbf{d}|}=\frac{-\mathrm{j} k}{4 \pi} \int d^{2} \hat{k} \mathrm{e}^{-j \mathbf{k} \cdot \mathbf{d}} \sum_{l=0}^{\infty}(-j)^{l}(2 l+1) h_{l}^{(2)}(k r) P_{l}(\hat{k} \cdot \hat{r})
$$

where the orders of summation and integration were interchanged. The speed-up of FMM is derived from the observation that the sum

$$
T_{L}(k r, \hat{k} \cdot \hat{r})=\sum_{l=0}^{L}(-j)^{l}(2 l+1) h_{l}^{(2)}(k r) P_{l}(\hat{k} \cdot \hat{r})
$$

is independent of kd and can thus be computed for various values of $k r$ which can be reused in the computation of the Green's function. The number of terms, $L+1$, kept for approximating the sum depends on the maximum value of $k d$, and the desired accuracy.

Noting that the direct path from the source to the field point can be decomposed into three parts (see Figure 11) with

$$
\mathbf{r}_{j i}=\mathbf{r}_{j m}+\mathbf{r}_{m m^{\prime}}-\mathbf{r}_{i m^{\prime}}
$$


the dyadic Green's function can be rewritten as

$$
\begin{aligned}
4 \pi \overline{\mathbf{G}}\left(\mathbf{r}_{j}, \mathbf{r}_{i}\right) & =\left[\overline{\mathbf{I}}-\frac{1}{k^{2}} \nabla \nabla^{\prime}\right] \frac{\mathrm{e}^{-\mathbf{j} k r_{j i}}}{r_{j i}} \\
& \approx \int d^{2} \hat{k}\left[\overline{\mathbf{I}}-\frac{1}{k^{2}} \nabla \nabla^{\prime}\right] \mathrm{e}^{-\mathbf{j k} \cdot\left(\mathbf{r}_{j m}-\mathbf{r}_{i m}\right)} T_{L}\left(k r_{m m^{\prime}}, \hat{k} \cdot \hat{r}_{m m^{\prime}}\right) \\
& =\int d^{2} \hat{k}[\overline{\mathbf{I}}-\hat{k} \hat{k}] \mathrm{e}^{-\mathbf{j k} \cdot\left(\mathbf{r}_{j m}-\mathbf{r}_{i m}\right)} T_{L}\left(k r_{m m^{\prime}}, \hat{k} \cdot \hat{r}_{m m^{\prime}}\right)
\end{aligned}
$$

The BI matrix entries can then be approximated as

$$
\begin{aligned}
\mathscr{B}_{i j} & =-2 k^{2} \int_{s} \mathrm{~d} s_{s} \widetilde{\mathbf{S}}_{i}(\mathbf{r}) \cdot \int_{s^{\prime}} \mathrm{d} s^{\prime}\left[\tilde{\mathbf{S}}_{j}\left(\mathbf{r}^{\prime}\right)-\frac{1}{k^{2}} \nabla^{\prime} \cdot \widetilde{\mathbf{S}}_{j}\left(\mathbf{r}^{\prime}\right) \nabla\right] \frac{\mathrm{e}^{-\mathrm{j} k R}}{R} \\
& \approx \frac{-\mathrm{j} k^{3}}{8 \pi} \int d^{2} \hat{k} \mathbf{V}_{f m j}(\hat{k}) \cdot T_{L}\left(k r_{m m^{\prime}}, \hat{k} \cdot \hat{r}_{m m^{\prime}}\right) \mathbf{V}_{s m^{\prime} i}^{*}(\hat{k})
\end{aligned}
$$

with

$$
\begin{aligned}
& \mathbf{V}_{s m^{\prime} i}(\hat{k})=\int_{S} \mathrm{~d} s^{\prime} \mathrm{e}^{-\mathbf{j} \mathbf{k} \cdot \mathbf{r}_{i m^{\prime}}}[\mathbf{I}-\hat{k} \hat{k}] \cdot \mathbf{S}_{i}\left(\mathbf{r}_{i}\right), \\
& \mathbf{V}_{f m j}(\hat{k})=\int_{S} \mathrm{~d} s \mathrm{e}^{-\mathbf{j} \mathbf{k} \cdot \mathbf{r}_{j m^{\prime}}}[\overline{\mathbf{I}}-\hat{k} \hat{k}] \cdot \mathbf{S}_{j}\left(\mathbf{r}_{j}\right)
\end{aligned}
$$

in which $\widetilde{\mathbf{S}}_{i}$ and $\widetilde{\mathbf{S}}_{j}$ denote the expansion and testing functions for the unknown magnetic surface current density. For our computations, we use the well known Rao-Wilton-Glisson [24] basis functions. Note also that '*' denotes complex conjugation.

To realize the FMM speed-up, the computational domain is divided into $M$ groups. The total memory storage needed is $\mathcal{O}\left(N^{2} / M\right)+\mathcal{O}(K N)+\mathcal{O}\left(K L M^{2}\right)$ where $K$ is the number of wave vector directions used in the numerical evaluation of the outermost integral in (48). Using the proportionalities $K \propto L^{2}, D^{2} \propto N / M$, and $L \propto D$, this expression can be simplified to $C_{1}\left(N^{2} / M\right)+$ $C_{2}(N M \sqrt{N / M})$, where $C_{1}$ and $C_{2}$ are machine (and implementation)-dependent constants. The coefficient $C_{2}$ is actually quite small compared to $C_{1}$ and thus the memory is dominated by the $\mathcal{O}\left(N^{2} / M\right)$ term.

The CPU requirement of this FMM implementation is $\mathcal{O}(N M)+\mathcal{O}\left(N^{2} / M\right)[16,19]$. This can be minimized by choosing $M=\sqrt{N}$ and the result is an $\mathcal{O}\left(N^{1.5}\right)$ algorithm. The memory required for the FMM also becomes $\mathcal{O}\left(N^{1.5}\right)$. In practice, both the operation cost and the memory requirement of FMM are less than those of standard MoM formulation for problem sizes larger than about 1000, making the FMM more suitable for the solution of large problems.

2.3.2. Results. One concern with the approximations introduced by FMM is accuracy for antenna simulations. Thus, for validation FMM was first applied for the analysis of 


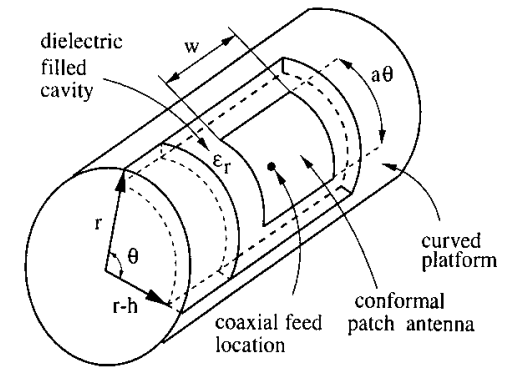

Figure 12. Microstrip patch antenna $(3.5 \mathrm{~cm} \times 3.5 \mathrm{~cm})$ on a cylindrical surface of radius $14.95 \mathrm{~cm}$. The patch is on a $0.3175 \mathrm{~cm}$ thick substrate having $\varepsilon_{r}=2.32$.

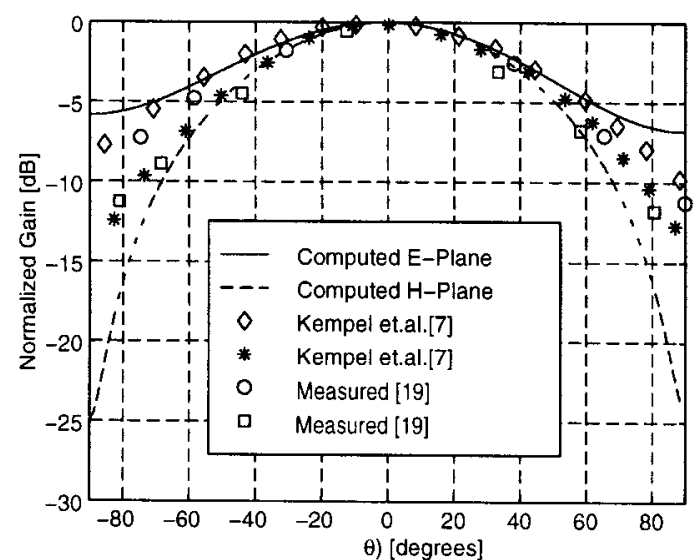

(a)

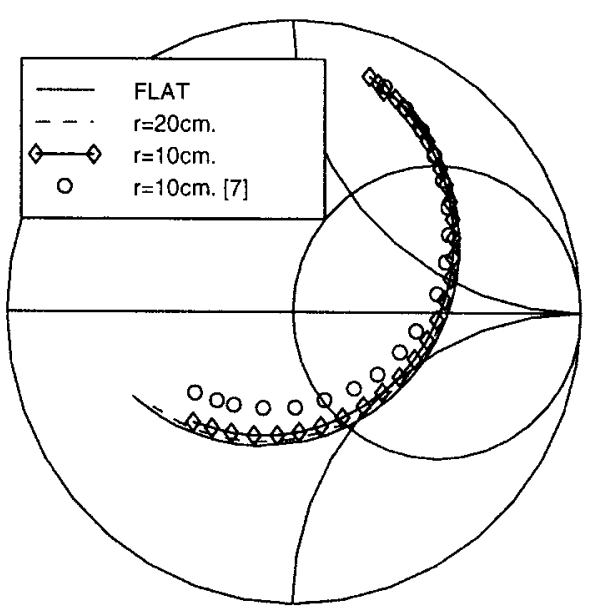

(b)

Figure 13. (a) Radiation pattern of the curved antenna in Figure 12, (b) input impedance for the circumferentially polarized antenna in Figure 12.

a $3.5 \mathrm{~cm} \times 3.5 \mathrm{~cm}$ cavity backed patch mounted on a cylindrical surface of radius $14.95 \mathrm{~cm}$. The patch is placed on the aperture of a cavity $0.3175 \mathrm{~cm}$ deep filled with a dielectric layer having $\varepsilon_{r}=2.32$. Figure 12 shows the layout of the curved antenna obtained by wrapping the flat antenna on the cylindrical surface and corresponds to the one considered in Reference [13].

As explained in Reference [13], two different radiation modes (axial and circumferential) exist depending on the feed location. The radiation patterns for the axial and circumferential excitation modes are shown in Figure 13(a). It is seen that the FMM calculations are in agreement with measurements in the broadside region, but the agreement deteriorates towards the shadow directions (around $\theta=-90^{\circ}$ and $\theta=90^{\circ}$ ), since the half-space Green's function is used, whereas in Reference [13] the exact cylindrical Green's function was employed. In the 


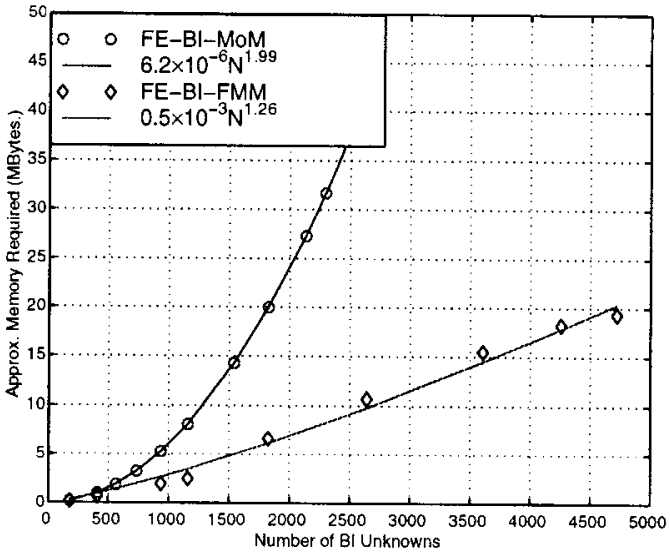

(a)

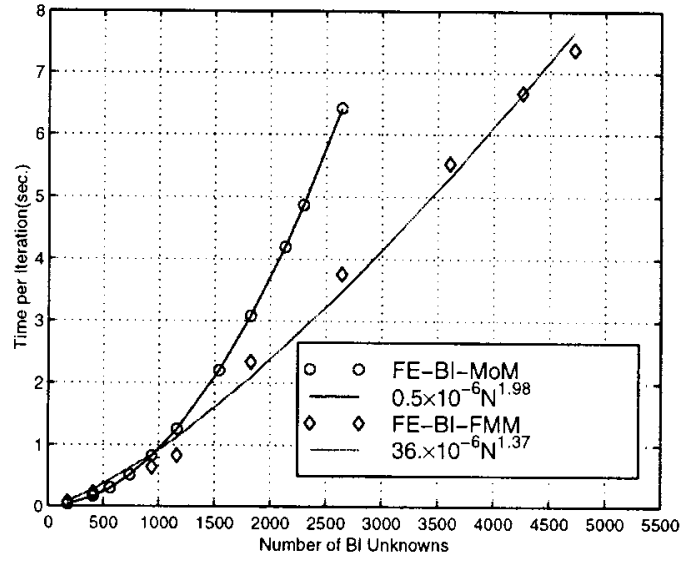

(b)

Figure 14. Memory (a) and CPU time (b) estimates of the FMM as a function of boundary integral unknowns.

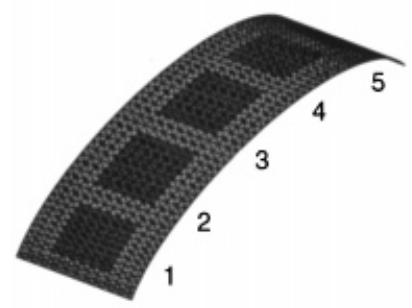

(a)

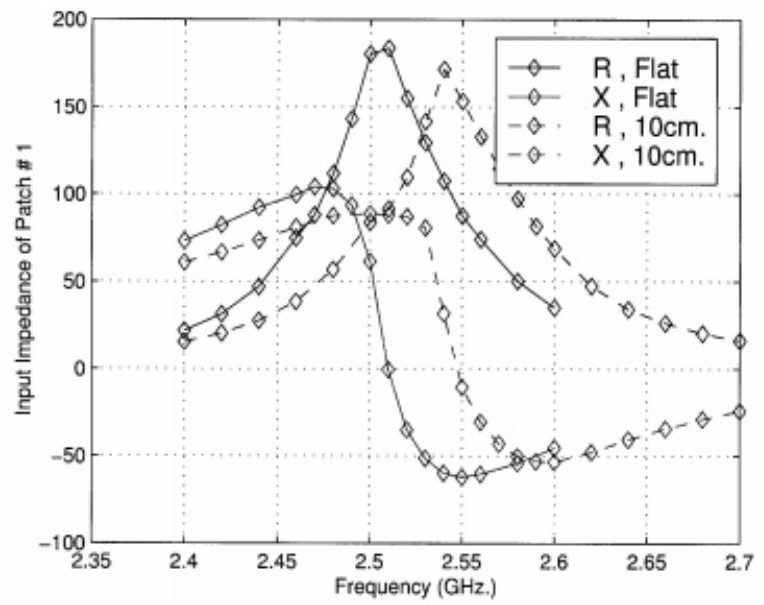

(b)

Figure 15. (a) Curved patch array geometry, (b) input impedance for patch element 1.

region $-60^{\circ} \leqslant \theta \leqslant 60^{\circ}$, the measured and calculated patterns agree to within $1 \mathrm{~dB}$. The input impedance dependence on antennas curvature is plotted in Figure 13(b) for the circumferentially polarized patch. The results are again in good agreement with those presented in Reference [13] and the resonant frequency can be accurately predicted since it is a more local phenomenon. Figure 14(a) and 14(b) show the comparison of the timings and approximate memory requirements for the conventional FE-BI method, with and without FMM. The complexity parameters 


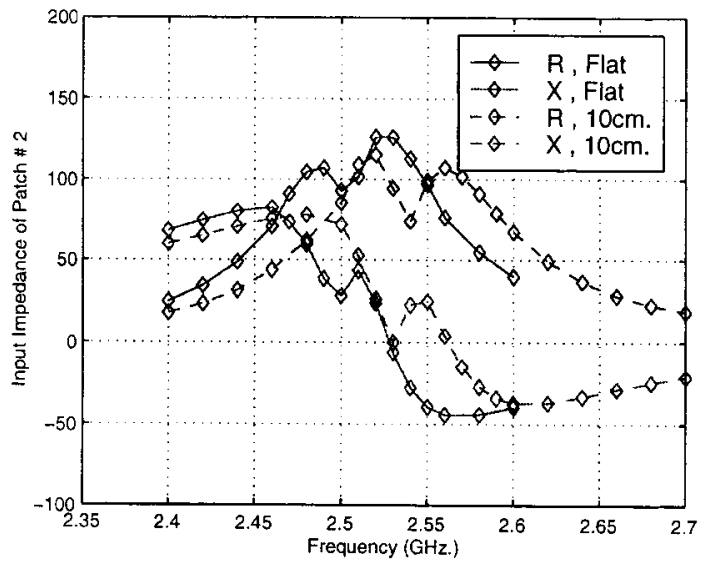

(a)

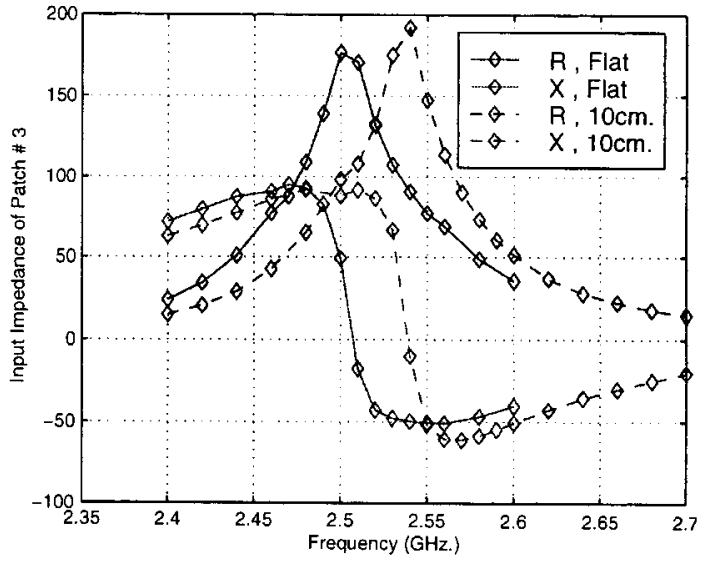

(b)

Figure 16. (a) Input impedance for patch element 2, (b) input impedance for patch element 3.

for the fitted curves are also given. Figure 14(a) depicts the trend for the total solution time indicating that the FMM is both robust and requires less resources for large-scale implementations.

Finite periodic arrays. The greatest advantage of the FMM implementation is its capability for an efficient computation of 3D problems. The necessity of using 3D-FFT's in AIM implementations may not render AIM as attractive without suitable optimizations on the implementation of the FFT. Thus, for doubly curved arrays we consider implementation of the FE-FI methods with FMM rather than AIM. Below, we show some results for the analysis of a five-element patch array on a curved surface. The geometrical configuration of the array is shown in Figure 15(a). A comparsion of the imput impedances of the individual patches for a flat and a curved array with a $10 \mathrm{~cm}$ radius of curvature is shown in Figures 15(b) and 16. An increased coupling between the different patches due to the curvature of the array can be especially seen in the input impedance of patch 2 .

\section{CONCLUSIONS}

The hybrid FE-BI method is a very powerful electromagnetic field calculation tool. Until recently, the large CPU time and memory complexity of the BI restricted the FE-BI method to moderately sized discretization models. In this paper, we considered speed-up and memory reductions of the FE-BI method by introducing fast integral methods to carry out the BI-related matrix-vector products. Among these methods, AIM has proven very attractive if planar BI termination surfaces are involved. In this case, only two-dimensional FFTs need to be performed and periodic Green's function can be included in a straightforward manner since they have the required convolutional property. On the other hand, the concept of FMM is basically three dimensional and, therefore, FMM is favourably used for non-planar termination surfaces. Both, 
AIM and FMM attain their speed-ups through an efficient (approximate) computation of the BI far interactions. However, since they keep the conventional BI formulation for the near-coupling terms they can produce results without any compromise in accuracy. In this section, AIM was applied to speed-up the BI termination of an FE-BI approach for infinite periodic structures. Also, a very efficient evaluation of the periodic Green's function series by virtue of the Ewald transformation was presented. Results were shown for the so-called photonic bandgap materials as well as frequency selective surfaces with commensurate and non-commensurate periodicities. Here, FMM was only used in conjunction with the modelling of antennas recessed in curved metallic platforms.

\section{REFERENCES}

1. Volakis JL, Özdemir T, and Gong J. Hybrid finite element methodologies for antennas and scattering. IEEE Transaction on Antennas and Propagation 1997; 493-507.

2. Gong J, Volakis JL, Wang HTG. Efficient finite element simulation of slot antennas using prismatic elements. Radio Science 1996; 31(6):1837-1844.

3. Özdemir T, Volakis JL. Triangular prisms for edge-based vector finite elements analysis. IEEE Transactions on Antennas Propagation 1997; 788-797.

4. Graglia RD, Wilton DR, Peterson AF, Gheorma I-L. Higher order interpolatory vector bases on prism elements. IEEE Transaction Antennas and Propagation 1998; 46(3):442-450.

5. Volakis JL, Chatterjee A, Kempel LC. Finite Element Methods for Electromagnetics. IEEE Press: Piscataway, NJ, 1998.

6. Lucas EW, Fontana TW. A 3-D hybrid finite element/boundary element method for the unified radiation and scattering analysis of general infinite periodic arrays. IEEE Transaction on Antennas and Propagation 1995; 43(2):145-153.

7. McGrath DT, Pyati VP. Periodic structure analysis using a hybrid finite element method. Radio Science 1996; 31(5):1173-1179.

8. Eibert TF, Volakis JL, Wilton DR, Jackson DR. Hybrid FE/BI modeling of 3D doubly periodic structures utilizing triangular prismatic elements and a MPIE formulation accelerated by the Ewald transformation. IEEE Transaction on Antennas and Propagation 1999; 47(5):843-849.

9. Rao SM, Wilton DR, Glisson AW. Electromagnetic scattering by surfaces of arbitrary shape. IEEE Transaction on Antennas and Propagation 1982; 30(3):409-418.

10. Yang H-YD, Diaz R, Alexopoulos NG. Reflection and transmission of waves from multilayer structures with planar implanted periodic material blocks. Journal of the Optic Society of America B 1997; 14(10):2513-2521.

11. Schmier RG. The artificial puck frequency selective surface. URSI Radio Science Meeting, Ann Arbor, 1993, p. 266.

12. Aroudaki H, Hansen V, Gemünd H-P, Kreysa E. Analysis of low-pass filters consisting of multiple stacked FSS's of different periodicities with applications in the submillimeter radioastronomy. IEEE Transaction on Antennas and Propagation 1995; 43(12):1486-1491.

13. Kempel LC, Volakis JL, Silva RJ. Radiation by cavity-backed antennas on a circular cylinder. IEE Proceedings of Microwave Antennas and Propagation 1995; 142(3):233-239.

14. Jordan KE, Richter GR, Sheng P. An efficient numerical evaluation of the Green's function for the Helmholtz operator on periodic structures. Journal of Computational Physics 1986; 43:222-235.

15. Ewald PP. Die Berechnung optischer und elektrostatischer Gitterpotentiale. Annals of Physics 1921; 64:253-268.

16. Chew WC, Jin J, Lu C, Michielssen E, Song JM. Fast solution methods in electromagnetics. IEEE Transaction on Antennas and Propagation 1997; 45(3):533-543.

17. Bleszynski E, Bleszynski M, Jaroszewicz T. AIM: adaptive integral method for solving large-scale electromagnetic scattering and radiation problems. Radio Science 1996; 31(5):1225-1251.

18. Coifman R, Rokhlin V, Wandzura S. The fast multipole method for the wave equation: a pedestrian prescription. IEEE Antennas and Propagation Magnetics 1993; 35:7-12.

19. Bindiganavale SS, Volakis JL. Comparison of three FMM techniques for solving hybrid FE-BI systems. IEEE Antennas and Propagation Magnetics 1993; 39(4):47-59.

20. Volakis JL, Barkeshli K. In Application of Iterative Methods to Electromagnetic and Signal Proceedings, Sarkar T (ed.). Elsevier: Amsterdam, 1991; 159-240. 
CONFORMAL ANTENNA AND ARRAY STRUCTURES UTILIZING FAST INTEGRAL METHODS 101

21. Bindiganavale SS. Fast memory-saving hybrid algorithms for electromagnetic scattering and radiation. Ph.D. thesis, University of Michigan, 1997.

22. Mittra R, Chan $\mathrm{CH}$, Cwik T. Techniques for analysing frequency selective surfaces - a review, Proceedings of the IEEE 1988; 76(12):1593-1615.

23. Abramowitz M, Stegun IA. Handbook of Mathematical Functions. National Bureau of Standards, 1972.

24. Rao SM, Wilton DR, Glisson AW. Electromagnetic scattering by surfaces of arbitrary shape. IEEE Transaction on Antennas and Propagation 1982; AP-30(3):409-418.

25. Sohtell EV. Microstrip antennas on a cylindrical surface. In Handbook of Microstrip antennas, James JR, Hall PS (eds). Pergrinus: London, 1989; 1227-1255. 